\title{
Exertional heat stroke with reversible severe cerebral edema
}

\author{
Sangkil Lee, Sung-Hyun Lee \\ Department of Neurology, Chungbuk National University College of Medicine, Cheongju, Korea
}

Severe cerebral edema associated with exertional heat stroke is a major cause of death or disability. However, few studies on severe cerebral edema resulting from heat stroke have reported neuroradiological findings. Moreover, all the patients in these previous reports either died or remained severely disabled. Here, we report a case of exertional heat stroke with severe cerebral edema that probably developed or worsened due to delayed body temperature normalization. In contrast to previous reports, the patient showed complete clinical and neuroradiological recovery. This rare case suggests that severe cerebral edema could be reversed through meticulous supportive management. Moreover, it confirms the importance of rapid and effective cooling in heat stroke treatment.

Keywords Heat stroke; Brain edema; Body temperature regulation; Cold water immersion

\section{INTRODUCTION}

Heat stroke is a multisystem condition that is characterized by central nervous system dysfunction and damage to other organs. It is associated with core body temperature (BT) $>40^{\circ} \mathrm{C}$. This can be caused by exposure to environmental heat (i.e., classic heat stroke) or strenuous physical exercise (i.e., exertional heat stroke). The main heat stroke treatments include rapid cooling and support of multiple organ functions. Despite modern intensive care, heat stroke is often fatal with many survivors sustaining functional impairment. ${ }^{1}$ Cerebral edema associated with heat stroke is a major cause of death or disability. ${ }^{2}$ However, few studies on severe cerebral edema resulting from heat stroke have reported neuroradiological findings. Moreover, all the patients described in these reports have either died or remained with severe disabilities. ${ }^{3.4}$ Here, we present

\section{Capsule}

Summary

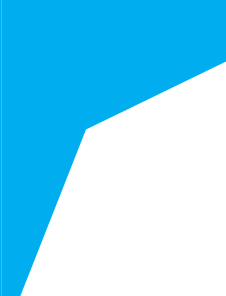

What is already known

Few studies on severe cerebral edema resulting from heat stroke have reported neuroradiological findings. Moreover, all the patients in these previous reports either died or remained severely disabled.

\section{What is new in the current study}

We report a case of exertional heat stroke with severe cerebral edema that probably developed or worsened due to delayed body temperature normalization but showed complete clinical and neuroradiological recovery through meticulous supportive management.
}

eISSN: 2383-4625

Received: 24 September 2019

Revised: 16 April 2020

Accepted: 27 April 2020

Correspondence to: Sung-Hyun Lee Department of Neurology, Chungbuk National University Hospital, 776 1 Sunhwan-ro, Seowon-gu, Cheongju 28644, Korea

E-mail: sunghlee@chungbuk.ac.kr ORCID

https://orcid.org/0000-0003-0598-8065

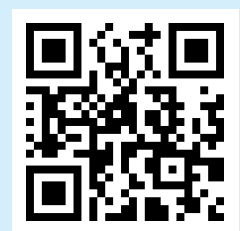


a patient with heat stroke who presented with severe cerebral edema and coma. This patient showed complete clinical and radiological recovery. The severe cerebral edema, which resulted in clinical deterioration, was probably caused by delayed BT normalization. The present case demonstrates the potential of reducing the incidence of death and disability associated with heat stroke. Moreover, this case report emphasizes the importance of rapid and effective cooling in heat stroke treatment.

\section{CASE REPORT}

On August 23, 2017, a 26-year-old obese (186 cm, $150 \mathrm{~kg}$, body mass index 43.4) man suddenly collapsed at 5 p.m. after about 7 hours of outdoor construction work in hot and humid weather conditions (maximum temperature and humidity of $32.4^{\circ} \mathrm{C}$ and $60 \%$, respectively). Informed consent was obtained from the patient for his anonymized information and clinical images to be published in this article. Before the incident, he was healthy and had worked at the site for about 3 weeks. He was brought to our emergency department (ED) at 5:30 p.m. in a deep coma state (Glasgow Coma Scale 3) with profuse perspiration. His pupils were equal and reactive; moreover, his heart rate $(\mathrm{HR})$, respiratory rate (RR), blood pressure (BP), and rectal BT were 174/min, 42/min, $90 / 60 \mathrm{mmHg}$, and $42^{\circ} \mathrm{C}$, respectively. A generalized convulsion episode occurred shortly after ED arrival. The laboratory findings were as follows: sodium, $132 \mathrm{mEq} / \mathrm{L} ;$ potassium, $4.4 \mathrm{mEq} / \mathrm{L}$; osmolality, $295 \mathrm{mOsml} / \mathrm{kg}$; creatinine, $1.9 \mathrm{mg} / \mathrm{dL}$; creatine kinase, $3,462 \mathrm{ng} / \mathrm{mL}$; aspartate aminotransferase (AST) and alanine aminotransferase (ALT), 93 and $46 \mathrm{U} / \mathrm{L}$; hemoglobin, $14.3 \mathrm{~g} / \mathrm{dL}$; and platelet, 195,000/ $\mu \mathrm{L}$. He was initially intubated and maintained on mechanical ventilation. Upon ED arrival (32 minutes after collapse), cooling through evaporative fanning, ice pack application, and intravenous cold saline infusion was initiated. After $10 \mathrm{~min}$ utes, an external cooling device with a target temperature of $33^{\circ} \mathrm{C}$ (Arctic Sun; Medivance, Louisville, CO, USA) was initiated. Subsequently, an additional external-cooling device (Blanketrol II; Cincinnati Sub-Zero Products, Cincinnati, OH, USA) was applied after 40 minutes. Bladder irrigation using $3 \mathrm{~L}$ of cold saline was completed thrice followed by injection of propacetamol and diclofenac. Three hours after collapsing, his BT remained at $40^{\circ} \mathrm{C}$ and his pupils became fixed (Glasgow Coma Scale 3). Further, his $H R, R R$, and BP were 128/min, 26/min, and 170/70 $\mathrm{mmHg}$, respectively. Five hours after collapsing, his BT dropped to $38.7^{\circ} \mathrm{C}$ and a brain computed tomography scan revealed severe cerebral edema (Fig. 1A). After the BT had lowered to $38^{\circ} \mathrm{C}$, only the external-cooling device was maintained for 63 hours with a target temperature of $36.5^{\circ} \mathrm{C}$ (Arctic Sun) where his BT ranged between

\section{$36.2^{\circ} \mathrm{C}$ to $37.3^{\circ} \mathrm{C}$}

On the second day, his systolic blood pressure decreased to 60 $\mathrm{mmHg}$ with accompanying oliguria and renal dysfunction (creatinine, $2.84 \mathrm{mg} / \mathrm{dL}$ ). Consequently, $5.0 \mathrm{~L}$ of $0.9 \%$ normal saline and $5 \%$ dextrose water solution was administered for 24 hours with central venous pressure monitoring and norepinephrine maintenance for 13 hours. His creatine kinase concentration increased to $9,527 \mathrm{ng} / \mathrm{mL}$. On the second day, continuous renal replacement therapy was initiated and maintained for the subsequent 8 days. The patient developed disseminated intravascular coagulation (platelet, 2,300/ $\mu \mathrm{L}$; international normalized ratio, 1.77; D-dimer, $7.6 \mu \mathrm{g} / \mathrm{mL}$; and fibrin degradation product, $19.7 \mu \mathrm{g} / \mathrm{mL}$ ) and received 8 units of fresh frozen plasma and 14 units of platelet concentrate for 48 hours. There was a gradual decrease of hemoglobin levels to $7.1 \mathrm{~g} / \mathrm{dL}$ on the 6th day; consequently, three units of packed red blood cells were administered. On the second day, empirical antibiotics with ceftriaxone and clindamycin were started and changed to ampicillin/sulbactam based on blood culture results showing methicillin-resistant coagulase-negative Staphylococcus epidermidis. Chest radiography revealed pulmonary congestion with pleural effusion between the 6th and 8th day. AST and ALT levels peaked on the second day (AST and ALT, 259 and $135 \mathrm{U} / \mathrm{L}$, respectively) while total bilirubin levels peaked $(7.37 \mathrm{mg} / \mathrm{dL})$ on the 6th day. Specific measures for treating cerebral edema were not implemented during the illness course. On the 5th day, the patient could localize to painful stimuli but did not open his eyes. After one week, the patient remained confused; however, he could obey simple commands and was successfully weaned from the ventilator. After 19 days, he was transferred to the general ward and showed complete recovery at 1 month after the onset. A brain computed tomography scan performed at 12 days showed complete resolution of the severe, whole-brain edema (Fig. 1B); the findings of a brain MRI scan performed at the 9th days after onset were normal (Fig. 1C); moreover, a brain computed tomography scan performed at the 12th days showed complete resolution of the severe, whole-brain edema (Fig. 1B). Throughout the 1-year follow-up, the patient remained completely normal.

\section{DISCUSSION}

Hyperthermia can cause blood-brain barrier disruption, which results in cerebral edema and neuronal damage. ' Cerebral edema has been reported in an autopsy series of patients who died from heat stroke and is considered as a major cause of death in this condition. ${ }^{2}$ However, few case reports have reported neuroimaging findings of severe cerebral edema resulting from heat stroke. 

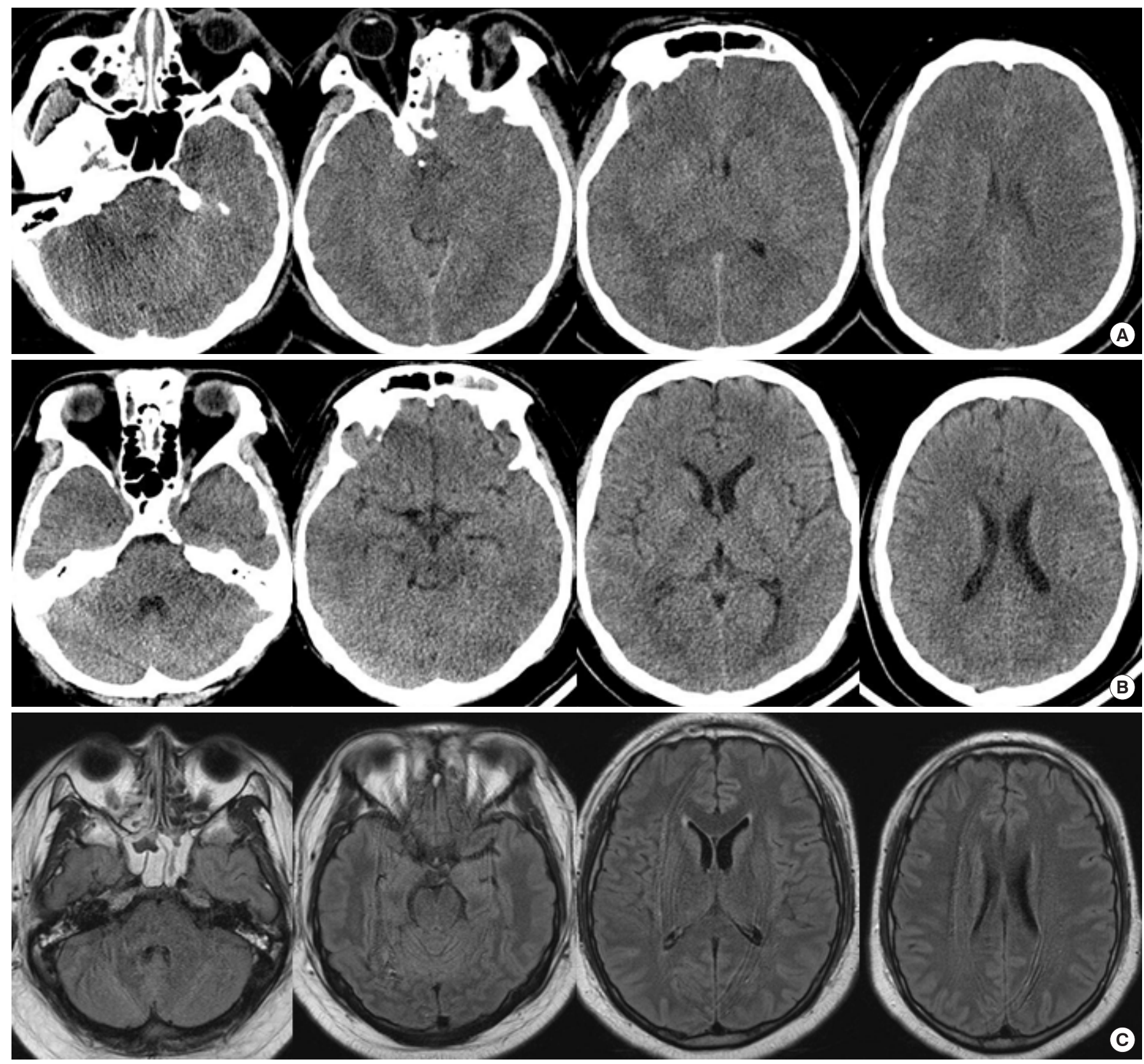

Fig. 1. Neuroimaging of this patient. (A) An initial brain computed tomography scan performed 5 hours after heat stroke onset showed diffuse severe cerebral edema. The basal cistern and ventricles are obliterated and the sulci are effaced. (B) A brain computed tomography scan performed at the 12th days after onset showed resolved severe cerebral edema. (C) Fluid-attenuated inversion recovery images obtained at the 9th days after onset revealed no abnormality. Informed consent for publication of the clinical images was obtained from the patient. Informed consent for publication of the clinical images was obtained from the patient.

Moreover, the patients in these reports subsequently died or had severe disabilities. ${ }^{3,4}$ To our knowledge, this is the first report of a patient with heat stroke who presented with severe cerebral edema and subsequently completely recovered. This may be a protean manifestation of heat stroke. The present case suggests that neuroimaging should be performed in case of deterioration in consciousness during the heat stroke treatment course. The ef- fective therapy for managing severe cerebral edema resulting from heat stroke remains unclear. Given that we did not include specific therapies of cerebral edema, including osmotherapy, corticosteroid, hypothermia, etc., meticulous supportive management could contribute toward alleviating cerebral edema in heat stroke.

Heat stroke treatment involves quickly lowering the BT with 
concomitant support to organ system functions. However, after full heat stroke onset, BT normalization may not prevent inflammation, coagulation, or progression to multiorgan dysfunction. ${ }^{1}$ Nonetheless, rapid and effective BT lowering is generally accepted as a mainstay of heat stroke treatment. The present patient was treated with various cooling methods. However, it took 5 hours for his BT to fall below $39^{\circ} \mathrm{C}$; moreover, 3 hours after collapsing, his initially reactive pupils became fixed. Further, there was a marked increase in his systolic blood pressure and a relative decrease in his heart rate when his BT was still $40^{\circ} \mathrm{C}$. These signs were indicative of cerebral edema development or aggravation after ED arrival.

Usually, in our ED, it takes $<3$ hours to lower the BT to below $39^{\circ} \mathrm{C}$ in patients with heat stroke using evaporative fanning, ice pack application, intravenous cold saline, and an external cooling device. However, in the present patient, who was extremely obese (body mass index 43.4), BT lowering to below $39^{\circ} \mathrm{C}$ took 5 hours. Consequently, we included bladder irrigation with cold saline, an additional external cooling device, and nonsteroidal anti-inflammatory drugs. Previously reported cases of heat stroke have taken about 5 hours or more to reach below $39^{\circ} \mathrm{C}^{3,5,6}$; moreover, another study reported taking $>2$ hours in 22/54 patients with heat stroke admitted to intensive care units.? These previous studies used various cooling measures except for cold water immersion, which is considered effective and safe, especially in patients with exertional heat stroke. ${ }^{8}$ Previous studies on patients with exertional heat stroke have reported that cold water immersion lowered the BT to below $39^{\circ} \mathrm{C}$ in less than 1 hour. ${ }^{9,10}$ However, the availability of a large water bath, difficulty in vital sign monitoring, as well as discomfort and agitation among patients, might limit the use of cold water immersion in the ED. ${ }^{8,11}$ At the time of the present case, the use of cold water immersion was not allowed in our ED, which might have made a difference in our patient. The use of cold water immersion in patients with exertional heat stroke might be considered, especially when the initial cooling rate of other methods is too slow due to individual physiological responses to hyperthermia and high variability of cooling methods.

Our case of exertional heat stroke is noteworthy since the patient developed very severe cerebral edema, which subsequently subsided without neurologic deficits. This is an extremely rare outcome and suggests that the initial deterioration in consciousness caused by cerebral edema might be reversed through meticulous supportive management. Moreover, this case emphasizes the importance of rapid, effective cooling in heat stroke treatment.

\section{CONFLICT OF INTEREST}

No potential conflict of interest relevant to this article was reported.

\section{REFERENCES}

1. Bouchama A, Knochel JP. Heat stroke. N Engl J Med 2002;346: 1978-88.

2. Goldstein LS, Dewhirst MW, Repacholi M, Kheifets L. Summa$r y$, conclusions and recommendations: adverse temperature levels in the human body. Int J Hyperthermia 2003;19:37384.

3. Herold J, Mitrasch A, Lorenz E, et al. Vomiting and collapse of a 28-year-old male long distance runner in middle European Summer. Internist (Berl) 2018;59:850-6.

4. Kuzume $D$, Inoue $S$, Takamatsu $M$, Sajima $K$, Kon-No Y, Yamasaki M. A case of heat stroke showing abnormal diffuse high intensity of the cerebral and cerebellar cortices in diffusion weighted image. Rinsho Shinkeigaku 2015;55:833-9.

5. Pease S, Bouadma L, Kermarrec N, Schortgen F, Regnier B, Wolff M. Early organ dysfunction course, cooling time and outcome in classic heatstroke. Intensive Care Med 2009;35:1454-8.

6. Parnell CJ, Restall J. Heat-stroke: a fatal case. Arch Emerg Med 1986:3:111-4.

7. Dematte JE, O'Mara K, Buescher J, et al. Near-fatal heat stroke during the 1995 heat wave in Chicago. Ann Intern Med 1998; 129:173-81.

8. Gaudio FG, Grissom CK. Cooling methods in heat stroke. J Emerg Med 2016;50:607-16.

9. Demartini JK, Casa DJ, Stearns R, et al. Effectiveness of cold water immersion in the treatment of exertional heat stroke at the Falmouth Road Race. Med Sci Sports Exerc 2015;47:240-5.

10. Divine JG, Daggy MW, Dixon EE, LeBlanc DP, Okragly RA, Hasselfeld KA. Case series of exertional heat stroke in runners during early spring: 2014 to 2016 Cincinnati Flying Pig Marathon. Curr Sports Med Rep 2018;17:151-8.

11. Lipman GS, Eifling KP, Ellis MA, et al. Wilderness Medical Society practice guidelines for the prevention and treatment of heat-related illness. Wilderness Environ Med 2013;24:351-61. 